\title{
Multiple Input Single Output Phase Retrieval
}

\author{
Yina Guo • Tao Wang • Jianyu Li . \\ Anhong Wang · Wenwu Wang
}

Received: date / Accepted: date

\begin{abstract}
In this paper, we consider the problem of recovering the phase information of multiple sources from a mixed phaseless Short-Time Fourier Transform (STFT) measurement, which is called multiple input single output (MISO) phase retrieval problem. It is an inherently ill-posed problem due to the lack of the phase and mixing information, and the existing phase retrieval algorithms are not explicitly designed for this case. To address the MISO phase retrieval problem, a least squares (LS) method coupled with an independent component analysis (ICA) algorithm is proposed for the case of sufficiently long window length. When these conditions are not met, an integrated algorithm is
\end{abstract}

Y. Guo

Dept. of Electronics and Information Engineering, Taiyuan University of Science and Technology, China

Tel.: +86-18636113711

E-mail: zulibest@163.com

T. Wang

Dept. of Electronics and Information Engineering, Taiyuan University of Science and Technology, China

E-mail: wangt@tyust.edu.cn

J. Li

Dept. of Electronics and Information Engineering, Taiyuan University of Science and Technology, China

E-mail: lijianyu@tyust.edu.cn

A. Wang

Dept. of Electronics and Information Engineering, Taiyuan University of Science and Technology, China

E-mail: wangty@tyust.edu.cn

W. Wang

Centre for Vision, Speech and Signal Processing, University of Surrey, UK

E-mail:w.wang@surrey.ac.uk 
presented, which combines a gradient descent (GD) algorithm by minimizing a non-convex loss function with an ICA algorithm. Experimental evaluation has been conducted to show that under appropriate conditions the proposed algorithms can explicitly recover the signals, the phases of the signals and the mixing matrix. In addition, the algorithm is robust to noise.

Keywords Multiple input single output (MISO) · phase retrieval · shorttime Fourier transform $(\mathrm{STFT}) \cdot$ non-convex optimization $\cdot$ independent component analysis (ICA)

\section{Introduction}

The problem of recovering a one-dimensional signal from its Fourier transform magnitude, known as phase retrieval, is of paramount importance in various engineering and scientific applications, such as X-ray crystallography [8,27], optics [11,34], astronomy [9,31], speech recognition [24,32, 38, 39], and blind channel estimation $[1,3]$. This problem has a long history and has been studied by many researchers $[5,7,10,12,14,18]$.

The phase retrieval problem originally arises from detectors that can sometimes only record the magnitude-square of the Fourier transform of a signal. Due to a lack of Fourier phase information, some forms of additional information are requiblack to identify the underlying signal efficiently. In this respect, the phase retrieval methods can be mainly classified into two categories based either on additional sparsity prior information $[10,12,18]$ or additional magnitude-only measurements (including structublack illuminations and masks $[5,7,14]$, and Short-Time Fourier Transform (STFT) magnitude-square measurements [4,21]). The key idea of using additional STFT magnitudesquare measurements is to introduce blackundancy in the magnitude-only measurements by maintaining a substantial overlap between adjacent short-time windows [21].

These phase retrieval methods have focused on recovering a single source from its Fourier transform magnitude. However, in certain cases, the problem of recovering multiple underlying sources from single mixed Fourier transform magnitudes, called multiple input single output (MISO) phase retrieval, is ever-present in CCD cameras and photosensitive films $[22,33,40]$, underwater acoustic signal detection [6,19], and speech enhancement [13,23]. This problem is ill-posed due to a lack of the phase property and the mixing information. The existing phase retrieval methods however can not provide a solution to the problem of recovering multiple underlying sources from single channel mixed Fourier transform magnitudes. Therefore, it is of great importance to study the problem of MISO phase retrieval.

Extending the study of Bendory and Eldar [4], we consider a closely-related problem of recovering the multiple underlying sources from single channel mixed STFT magnitude-square measurements. In this work, our contribution is three-fold: 
(1) MISO phase retrieval model: A new model of the MISO phase retrieval problem is proposed in order to recover multiple underlying sources from single channel mixed STFT magnitude-square measurements, corrupted by noise.

(2) MISO phase retrieval algorithms: Due to the absence of Fourier phase information and mixed information, we explore hybrid methods by introducing additional STFT magnitude-square measurements as well as estimating the mixing information. For maximal overlap between adjacent windows, the drop size $(L=1)$ and sufficiently long window length $W$, the solution can be constructed as an improved least square (LS) method coupled with an independent component analysis (ICA) algorithm, called LS-ICA. When these conditions are not met, an integrated algorithm called GD-ICA is proposed, which combines a gradient descent (GD) algorithm by minimizing a non-convex loss function with an ICA algorithm. According to different conditions, the mixed signal can be recoveblack either by the LS-ICA algorithm or the GD-ICA algorithm. The residual offeblack by the mixed phaseless STFT measurement and the recoveblack mixed signal can be used to construct another mixed signal. Then we use an ICA algorithm to estimate the mixing information and the source signals from the constructed mixed signals.

(3) Initialization of the GD algorithm: It is shown in [4] that the initialization of the GD algorithm can be equivalently posed as a constrained LS problem. However, it is prone to over-fitting with less training data, and requires the rank restriction of the regression variable. To address this issue, we propose to use the principle eigenvector of a designed correlation matrix to initialize the GD-ICA algorithm that minimizes a non-convex loss function. The principle eigenvector of the designed correlation matrix can be obtained by a LS solution with a penalty term. The new loss function may provide significant benefits in two aspects. First, it prevents over-fitting and improves generalization performance. Second, it relaxes the rank restriction of regression variable.

The paper is organized as follows. Section 2 describes the background, i.e., the method by Bendory and Eldar [4]. Section 3 formulates a mathematical model and gives the assumptions for the problem of MISO phase retrieval from single channel mixed STFT magnitude-square measurement. Section 4 discusses the uniqueness of the MISO phase retrieval problem and presents the conditions under which it has a solution by combining an improved LS method with an ICA algorithm. When these conditions are not met, an integrated algorithm is proposed which couples a GD algorithm by minimizing a nonconvex loss function with an ICA algorithm. This section also explores the initialization method for the GD-ICA algorithm. Section 5 shows numerical experimental results. Section 6 concludes the paper and draws potential future research directions.

Notation: Boldface small and capital letters denote vectors (e.g. $\mathbf{x}$ ) and matrices (e.g. $\mathbf{X}$ ), respectively. The superscripts ${ }^{T},{ }^{*}$ and ${ }^{\dagger}$ denote the transpose, Hermitian, and Moore-Penrose pseudo-inverse of a matrix, respectively. $\circ$ means the Hadamard (element-wise) product operator. $\operatorname{tr}(\cdot)$ takes the trace of 


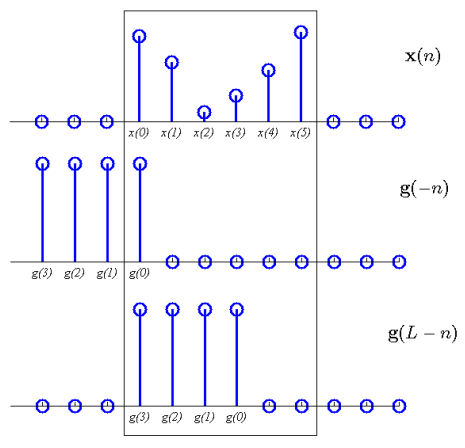

(a)

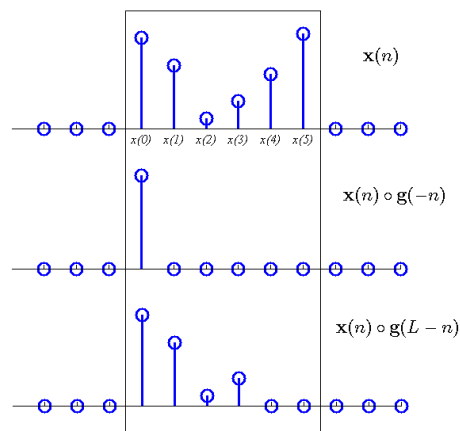

(b)

Fig. 1: A shifting example of the sliding window overlapped with the signal $(R=\lceil N / L\rceil=2)$ for $N=6, W=4$ and $L=3$.

a matrix. The $l$ th circular diagonal element of a matrix is denoted by $\operatorname{diag}(\cdot, l)$. $\mathrm{k}(\cdot)$ takes the kurtosis of the argument.

\section{Background}

Recently, the problem of recovering phase information of an underlying signal from the phaseless STFT measurement has been studied in [4], as discussed next.

The STFT X of a one-dimensional (1D) signal is defined as the Fourier transform of the signal $\mathbf{x} \in \mathbb{C}^{N}$ multiplied by a real sliding window $\mathbf{g}$ of length $2 \leq W \leq N$.

$$
X(\tau, k)=\sum_{n=0}^{N-1} x(n) g(\tau L-n) e^{-2 j \pi k n / N},
$$

for $\tau=0, \ldots, R-1, k=0, \ldots, N-1$, where $L$ depicts the maximal overlapping between adjacent windows, $R=\lceil N / L\rceil$ denotes the number of short-time windows consideblack, and \lceil\rceil rounds the argument to the smallest integer that is not less than the argument.

Fig. 1 shows an example $\mathbf{g}_{\tau L}=\{g(\tau L-n)\}_{n=0}^{N-1}$ and applying it to a signal by shifting the sliding window $\mathbf{g}$ by $\tau L$ time units. $\mathbf{x}$ and $\mathbf{g}$ are zero-padded over the boundaries of (1). The $\tau$ th row of $\mathbf{X}$ corresponds to the $N$-point DFT of $\mathbf{x} \circ \mathbf{g}_{\tau L}$.

Let $\mathbf{Z}$ be an $N \times R$ measurement matrix corresponding to the STFT magnitude-square of the underlying signal $\mathbf{x}$.

$$
\mathbf{Z}=|\mathbf{X}|^{2} \text {. }
$$

The purpose of the algorithms in [4] is to estimate $\mathbf{x}$ from $\mathbf{Z}$. 
The key idea is to introduce blackundancy in the magnitude-only measurements by maintaining a substantial overlap between adjacent short-time windows [7].

(1) For sufficiently long window length, the solution can be constructed as a least-squares (LS) method.

(2) When the window length is not long enough, a gradient descent (GD) algorithm is used to solve the phase retrieval problem.

Experiments show that the algorithms in [4] can exactly recover $\mathbf{x}$ from $\mathbf{Z}$ under certain conditions.

In order to simplify the quadratic system of equations and reveal the underlying structure, the problem of STFT phase retrieval can be equivalently stated in terms of the short-time autocorrelation. The algorithms in [4] begin by taking the DFT of the phaseless STFT measurement (2), as follows

$$
\begin{aligned}
& Y(\tau, l)=\frac{1}{N} \sum_{k=0}^{N-1} Z(\tau, k) e^{-2 j \pi k l / N} \\
= & \sum_{n=0}^{N-1} x(n) x^{*}(n+l) g(\tau L-n) g(\tau L-n-l),
\end{aligned}
$$

where $Y(\tau, l)$ is equal to zero for all $\tau$ when $W \leq l \leq(N-W)$ and can be interpreted as a " $W$ bandlimited" function. The DFT is normalized by $1 / N$. For fixed $\tau, \mathbf{Y}$ can be seen as the autocorrelation of $\mathbf{x} \circ \mathbf{g}_{\tau L}$ [4]. Note that the $\tau$ th row of $\mathbf{Z}$ and the $\tau$ th row of $\mathbf{Y}$ are Fourier pairs. Hence, for a particular $\tau$, if $Z(\tau, l)$ for $0 \leq l \leq N-1$ is available, then $Y(\tau, l)$ for $0 \leq l \leq N-1$ can be calculated by taking an inverse Fourier transform [21].

Theorem 2.1: $Z(\tau, k)$ for $0 \leq k \leq 2 W-2$ is sufficient to calculate $Y(\tau, l)$ for $0 \leq l \leq N-1$.

(1) For $W \geq\lceil(N+1) / 2\rceil$ and $L=1$, the problem of recovering $\mathbf{x}$ from the measurement $\mathbf{Z}$ can therefore be equivalently posed as a constrained LS loss function derived from (3). With the quadratic constraint, (3) is equivalent to a non-convex problem.

$$
\min _{\mathbf{x} \in \mathbb{C}^{N}} \sum_{l=-(W-1)}^{W-1}\left\|\mathbf{y}_{l}-\mathbf{G}_{l} \operatorname{diag}(\mathbf{X}, l)\right\|_{2}^{2},
$$

subject to $\mathbf{X}=\mathbf{x x}^{*}$,

where $\mathbf{y}_{l}=\{Y(\tau, l)\}_{\tau=0}^{R-1}$, the $(\tau, n)$ th entry of the matrix $\mathbf{G}_{l} \in \mathbb{R}^{R \times N}$ is given by $g(\tau L-n) g(\tau L-n-l), \mathrm{P}_{-l}=\mathrm{P}_{l}^{T}$, and $\left(\mathrm{P}_{l} x\right)(n)=x(n+l)$. Then the first column of $\mathbf{G}_{l}$ can be given by the non-vanishing matrix $\mathbf{g} \circ\left(\mathbf{P}_{-l} \mathbf{g}\right)$, where $\mathbf{g}=$ $\{g(n)\}_{n=0}^{N-1} \cdot \mathbf{G}_{l}$ as a circulant matrix can be factoblack as $\mathbf{G}_{l}=\mathbf{F}^{*} \boldsymbol{\Sigma}_{l} \mathbf{F}$, where $\mathbf{F}$ is the DFT matrix and $\boldsymbol{\Sigma}_{l}$ is a diagonal matrix (as in [4]). For sufficiently long window length, [4] shows that the LS algorithm is effective for recovering $\mathbf{x}$ from $\mathbf{Z}$. 
Theorem 2.2: Let $L=1$. Suppose that $\mathbf{x}$ is non-vanishing and the DFT of $\mathbf{g} \circ\left(\mathbf{P}_{-l} \mathbf{g}\right)$ is non-vanishing for $l=0,1$. Then $\mathbf{Z}$ determines $\mathbf{x}$ uniquely (up to a global phase).

Theorem 2.3: Let $L=1$. Suppose that $\mathbf{g}$ is an admissible window (rectangular window) of length $W \geq\lceil N+1 / 2\rceil$. The DFT of $\mathbf{g} \circ\left(\mathbf{P}_{-l} \mathbf{g}\right)$ is nonvanishing for $l=-(W-1), \ldots, W-1$ and $\mathbf{G}_{l}$ is invertible. Then (4) recovers any complex signal uniquely (up to a global phase) and efficiently.

(2) In some cases, the window is shorter than or equal to $\lceil N / 2\rceil$, (4) does not have a similar LS solution. In these cases, a GD algorithm is used to recover the signal by minimizing a non-convex loss function. In order to initialize the GD algorithm, the same LS-based algorithm is used. However, for $l=W, \ldots,(N-W), \operatorname{diag}(\mathbf{X}, l)$ can not be estimated as the elements in the matrices $\mathbf{G}_{l}$ are zero. It was proved in [4] that the principle eigenvector of the approximation matrix $\mathbf{X}_{0}$ can be a good estimate of $\mathbf{x}$ with appropriate normalization.

For $2 \leq W \leq\lceil N / 2\rceil$, let $\mathbf{D}_{\tau L} \in \mathbb{R}^{N \times N}$ be a diagonal matrix composed of the entries of $\mathbf{g}_{\tau L}$, a non-convex loss function is formed from (3) for recovering $\mathbf{x}$ from $\mathbf{Z}[4]$.

$$
f(\mathbf{x})=\frac{1}{2} \sum_{\tau=0}^{R-1} \sum_{l=-(W-1)}^{W-1}\left(\mathbf{x}^{*} \mathbf{H}_{\tau, l} \mathbf{x}-Y(\tau, l)\right)^{2},
$$

where $\mathbf{H}_{\tau, l}=\mathrm{P}_{-l} \mathbf{D}_{\tau L} \mathbf{D}_{\tau L-l}, \mathbf{x}^{*} \mathbf{H}_{\tau, l} \mathbf{x}=\operatorname{tr}\left(\mathbf{X} \mathbf{H}_{\tau, l}\right)$.

A GD algorithm is adopted to recover $\mathbf{x}$ by minimizing (5). If $\mathbf{g}$ is not long enough, [4] indicates that the GD algorithm can recover $\mathbf{x}$ from $\mathbf{Z}$ effectively.

Loss function for initialization: In the GD algorithm, the geometry of the loss function for the initialization has heavy effects on the properties of the algorithm. In [4], (4) is used to determine the initialization.

Theorem 2.4: Let $L=1$. Suppose that $\mathbf{x} \in \mathbb{C}_{1 / \sqrt{N}}^{N}$ and $\mathbf{g}$ is an admissible window of length $W \geq 2$. For $l=0,1, \ldots, W-1, \mathbf{X}_{0}$ is defined as a matrix obeying

$$
\operatorname{diag}\left(\mathbf{X}_{0}, l\right)=\left\{\begin{array}{lc}
\mathbf{G}_{l}^{-1} \mathbf{y}_{l}, & l=0, \ldots, W-1, \\
0, & \text { otherwise }
\end{array}\right.
$$

where $\mathbf{y}_{l}, \mathbf{G}_{l}$ are defined as in (4).

If $L=1$, the initialization $\mathbf{x}_{0}$ can be determined by (4) directly.

In the case of $L>1, \mathbf{y}_{l}=\{Y(\tau, l)\}_{\tau=0}^{R-1}$ has some missing entries. The up-sampling version $\tilde{\mathbf{y}}_{l}$ can be obtained by expansion and interpolation.

Theorem 2.5: Let $\tilde{\mathbf{g}}=\{g[(-n) \bmod N]\}_{n=0}^{N-1}$. Suppose that $\tilde{\mathbf{g}} \in \mathbb{R}^{N}$ is an ideal low-pass filter with bandwidth $R . \mathbf{y}=\mathbf{G x}$, where $\mathbf{G}$ is a circulant matrix whose first column is $\tilde{\mathbf{g}}$. Let $\mathbf{y}_{L}=\left\{Y_{L}(\tau, l)\right\}_{n=0}^{R-1}$ be a down-sampled version. 


$$
\begin{aligned}
& Y_{L}(n, l)=\left\{\begin{array}{lc}
Y_{L}(\tau, l), & n=\tau L, \\
0, & \text { otherwise, }
\end{array}\right. \\
& \text { Let } \hat{\mathbf{y}}_{L}=\left\{Y_{L}(n, l)\right\}_{n=0}^{N-1} \text { for fixed } l, \\
& \tilde{\mathbf{y}}=\left(\mathbf{F}_{p}^{*} \mathbf{F}_{p}\right)_{\mathbf{y}_{L},}
\end{aligned}
$$

where $\mathbf{F}_{p}$ is a partial Fourier matrix consisting of the first $R$ rows of the DFT matrix $\mathbf{F}$ defined as in (4).

\section{Mathematical Model and Assumptions}

Consider a collaborative estimation task that is induced by multi-source phase retrieval from single channel mixed phaseless STFT measurement in a noisy environment. We present a mathematical model and the assumptions for this collaborative estimation task in this section.

3.1 Model for the MISO phase retrieval problem

The multiple underlying sources are $\mathbf{S}=\left[\mathbf{s}_{1}, \mathbf{s}_{2}, \ldots, \mathbf{s}_{M}\right]^{T} \in \mathbb{C}^{M \times N}$. A linear mixture of multiple underlying sources is defined as $\mathbf{x}=\mathbf{a S}$, where $\mathbf{x} \in \mathbb{C}^{N}$ is the mixture and the mixing vector $\mathbf{a}=\left[a_{1}, a_{2}, \ldots, a_{M}\right] \in \mathbb{R}^{M}[2,30,35-37]$.

The STFT $\mathbf{X} \in \mathbb{C}^{N}$ of the mixed signal $\mathbf{x}$ can be defined as

$$
X(\tau, k)=\sum_{n=0}^{N-1} x(n) g(\tau L-n) e^{-2 j \pi k n / N}
$$

where $\mathbf{g}, \tau, k, R$ and $L$ are interpreted in (1).

Let $\mathbf{Z}$ be a $R \times N$ measurement corresponding to the STFT magnitudesquare $|\mathbf{X}|^{2}$ of the mixed signal $\mathbf{x}$ corrupted by noise.

$$
\mathbf{Z}=|\mathbf{X}|^{2}+\mathbf{B},
$$

where $\mathbf{B}$ is a random $R \times N$ matrix which represents noise, following the standard normal distribution with zero mean and unit variance.

The model of MISO phase retrieval problem is illustrated in Fig. 2. The aim is to recover the underlying sources $\mathbf{S}$ from the phaseless STFT measurement $\mathbf{Z}$, corrupted by noise $\mathbf{B}$. 


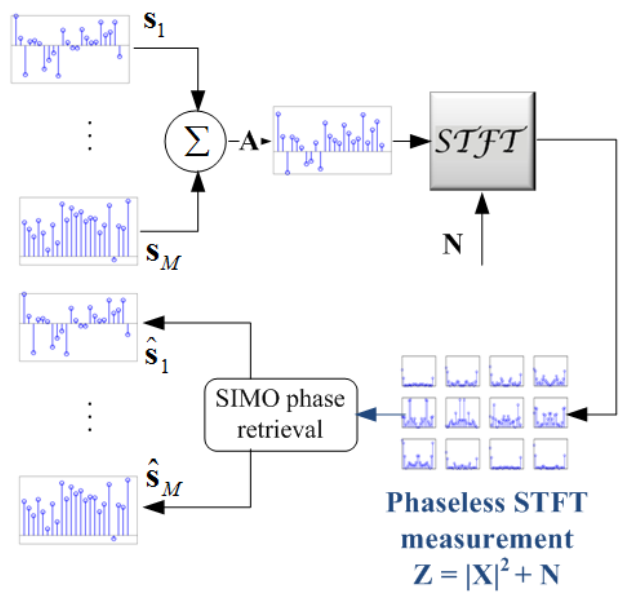

Fig. 2: Model for MISO phase retrieval.

\subsection{Assumptions for MISO phase retrieval}

To address the MISO phase retrieval problem, two assumptions are utilized for constructing the MISO phase retrieval model:

(i) The mixed signal $\mathbf{x}$ is a linear mixture of the multiple underlying source signals $\mathbf{S}$ multiplied by the mixing matrix $\mathbf{A}$.

(ii) The multiple underlying sources $\mathbf{S}$ are as independent as possible of each other.

\section{MISO Phase Retrieval Algorithms}

In this section, we discuss the conditions for solving the MISO phase retrieval problem and under which we provide a three-step solution. The first step is to recover the mixed signal $\mathbf{x}$ from its mixed phaseless STFT measurement $\mathbf{Z}$. The second step is to construct multiple mixed signals $\mathbf{X}=\left[\mathbf{x}_{1}, \mathbf{x}_{2}, \ldots, \mathbf{x}_{M}\right]^{T}$ by the residuals provided from the multiple constructed mixed phaseless STFT measurements $\mathbf{K}=\left[\mathbf{Z}_{0}, \mathbf{Z}_{1}, \ldots, \mathbf{Z}_{M-1}\right]^{T}$. The last step is to recover multiple underlying sources $\hat{\mathbf{S}}$ from the constructed mixed signals $\mathbf{X}$.

Fig. 3 illustrates the overview of the solution. Let $\mathbf{Z}_{0}=\mathbf{Z}$, we take $i=1$ and $M=2$ as an example. The first mixed signal $\mathbf{x}_{1}$ is recoveblack from the mixed phaseless STFT measurement $\mathbf{Z}_{0}$. The residual $\mathbf{Z}_{1}$ offeblack by $\mathbf{Z}_{0}$ and $\mathbf{x}_{1}$ can be used to construct another mixed signal $\mathbf{x}_{2}$ when its magnitude is compensated by $C_{1}$ which is defined as in (11) shown in the next section. Until all the mixed signals $\mathbf{X}=\left[\mathbf{x}_{1}, \mathbf{x}_{2}\right]^{T}$ have been constructed, we can recover the multiple underlying sources $\hat{\mathbf{S}}=\left[\hat{\mathbf{s}}_{1}, \hat{\mathbf{s}}_{2}\right]^{T}$ and estimate the mixing matrix $\mathbf{A}$.

For $L=1$ and sufficiently long window length $W$, the solution can be constructed as an improved LS method coupled with an ICA algorithm, called LS- 


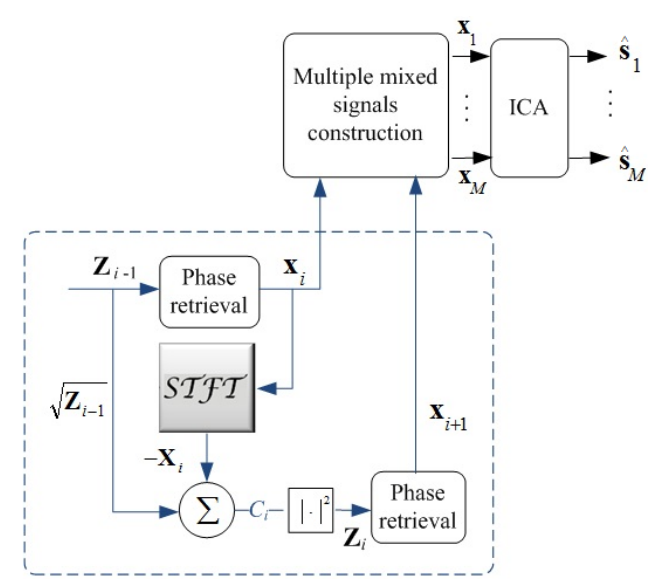

Fig. 3: Overview of the solution for the MISO phase retrieval problem. For $i=1, \mathbf{Z}_{0}$ is the single-channel mixed phaseless STFT measurement. The first mixed signal $\mathbf{x}_{1}$ and its phase are recoveblack from $\mathbf{Z}_{0}$. The residual $\mathbf{Z}_{1}$ is obtained by $\mathbf{Z}_{0}$ and $\mathbf{x}_{1}$. Then another mixed signal $\mathbf{x}_{2}$ and its phase can be constructed from $\mathbf{Z}_{1}$. From the constructed mixed signals $\mathbf{X}=\left[\mathbf{x}_{1}, \mathbf{x}_{2}\right]^{T}$, the multiple underlying sources $\hat{\mathbf{S}}=\left[\hat{\mathbf{s}}_{1}, \hat{\mathbf{s}}_{2}\right]^{T}$ can be estimated by ICA.

ICA. When these conditions are not met, an integrated algorithm is proposed which combines a GD algorithm by minimizing a non-convex loss function with an ICA algorithm.

\subsection{LS-ICA algorithm}

The fundamental questions of MISO phase retrieval are whether the multiple mixed signals $\mathbf{X}$ can be constructed from the quadratic measurement operator of $\mathbf{Z}$ and whether the underlying signals $\mathbf{S}$ can be determined uniquely from $\mathbf{X}$. Based on the study of Eldar et al. [4], we propose an LS-ICA algorithm which is effective to solve this problem for for the maximal overlapping between adjacent windows $L=1$ and sufficiently long window length $W$.

(1) Recovery of the phases of the mixed signal $\mathbf{x}$

For the purpose of constructing multiple mixed phaseless STFT measurements, we first recover the mixed signal $\mathbf{x}$. To simplify the quadratic system of equations and obtain requiblack correlation data [26], we take DFT to the phaseless STFT measurement $\mathbf{Z}$. Let $\mathbf{Z}_{0}=\mathbf{Z}, \mathbf{x}_{1}=\mathbf{x}$, for $i=1, \cdots, M$,

$$
\begin{aligned}
& Y_{i-1}(\tau, l)=\frac{1}{N} \sum_{k=0}^{N-1} Z_{i-1}(\tau, k) e^{-2 j \pi k l / N} \\
& =\sum_{n=0}^{N-1} x_{i}(n) x_{i}^{*}(n+l) g(\tau L-n) g(\tau L-n-l),
\end{aligned}
$$


where $Y_{i-1}(\tau, l)$ can be expressed as a band-limited function for $W \leq l \leq$ $(N-W)$. For fixed $\tau, \mathbf{Y}_{i-1}$ can be seen as the autocorrelation of $\mathbf{x}_{i} \circ \mathbf{g}_{\tau L}$, where $\mathbf{g}_{\tau L}$ is defined as in (1).

For $W \geq\lceil(N+1) / 2\rceil$ and $L=1$, it was shown in [4] that (3) can be equivalently posed as a constrained LS problem as (4). However, this method is prone to over-fitting with less training data and requires the rank restriction for $\mathbf{G}_{l}$. To address this issue, a penalty term is introduced as follows [17],

$$
\begin{aligned}
& \min _{\mathbf{x}_{i} \in \mathbb{C}^{N}} \sum_{l=-a}^{a}\left\|\mathbf{y}_{(i-1) l}-\mathbf{G}_{l} \operatorname{diag}\left(\mathbf{F}_{i}, l\right)\right\|_{2}^{2}+\lambda\left\|\operatorname{diag}\left(\mathbf{F}_{i}, l\right)\right\|_{2}^{2}, \\
& \text { subject to } \mathbf{F}_{i}=\mathbf{x}_{i} \mathbf{x}_{i}^{*},
\end{aligned}
$$

where $a=W-1, \lambda$ is the regularization coefficient, $\mathbf{y}_{(i-1) l}$ and $\mathbf{G}_{l}$ are defined as in (4). The modification of (4) may provide significant benefits in two aspects. First, it prevents over-fitting and improves generalization performance. Second, it relaxes the rank restriction of $\mathbf{G}_{l}$.

A matrix $\mathbf{F}_{i}$ is constructed from (8) as follows [17]

$$
\operatorname{diag}\left(\mathbf{F}_{i}, l\right)=\left\{\begin{array}{cl}
\left(\mathbf{G}_{l}^{T} \mathbf{G}_{l}+\lambda I\right)^{-1} \mathbf{G}_{l}^{T} \mathbf{y}_{(i-1) l}, & l=-a, \ldots, a, \\
0, & \text { otherwise },
\end{array}\right.
$$

where $\mathbf{x}_{i}$ is a principle eigenvector of $\mathbf{F}_{i}$.

From (7) to (9), $\mathbf{x}_{i}$ can be recoveblack from $\mathbf{Z}_{i-1}$. In order to remove the magnitude effect of the mixing matrix, the recoveblack mixed signal is normalized as $\mathbf{x}_{i}$.

(2) Construction of multiple mixed signals $\mathbf{X}$

In order to construct multiple mixed signals, we consider to construct multiple mixed phaseless STFT measurements. For $i=1, \cdots, M$, we use the residual to construct the $i$ th phaseless STFT magnitude-square measurement, which is defined as

$$
\mathbf{Z}_{i}=\left|C_{i}\left[\sqrt{\mathbf{Z}_{i-1}}-\mathbf{X}_{i}\right]\right|^{2}
$$

where

$$
C_{i}=\left\|\sqrt{\mathbf{Z}_{i-1}}\right\|_{2} /\left\|\sqrt{\mathbf{Z}_{i-1}}-\mathbf{X}_{i}\right\|_{2},
$$

represents the magnitude compensation and $\mathbf{X}_{i}$ means the STFT of $\mathbf{x}_{i}$. For each $\mathbf{Z}_{i-1}$, the above procedures from (7) to (9) are repeated until all the mixed signals have been recoveblack from the constructed phaseless STFT measurements $\mathbf{K}=\left[\mathbf{Z}_{0}, \ldots, \mathbf{Z}_{M-1}\right]^{T}$ and normalized as $\mathbf{X}=\left[\mathbf{x}_{1}, \ldots, \mathbf{x}_{M}\right]^{T}$.

(3) Recovery of multiple underlying sources $\hat{\mathbf{S}}$

All the normalized mixed signals $\mathbf{X}$ are used as the input for the recovery of the underlying sources. On the basis of the independent component analysis idea $[15,16,20]$, we use the Complex Maximization of Nongaussianity (CMN) algorithm presented in $[28,29]$, which is an effective algorithm for both circular and non-circular sources using complex functions.

The whitening mixed signal $\tilde{\mathbf{x}}_{i}=\mathbf{U x}_{i}$ are transformed by a whitening matrix $\mathbf{U}[28]$. Here $E\left\{\tilde{\mathbf{x}}_{i} \tilde{\mathbf{x}}_{i}^{*}\right\}=\mathbf{I}$. 
The use of whitening allows us to search for an orthogonal matrix $\mathbf{W}$ as $E\left\{\mathbf{W} \tilde{\mathbf{x}}_{i}\left(\mathbf{W} \tilde{\mathbf{x}}_{i}\right)^{*}\right\}=\mathbf{W} E\left\{\tilde{\mathbf{x}}_{i} \tilde{\mathbf{x}}_{i}^{*}\right\} \mathbf{W}^{*}=\mathbf{I}$.

Each source $s_{k}$ is estimated by finding a vector $\mathbf{w}$ such that

$$
s_{i k}=\mathbf{w}_{k}^{*} \tilde{\mathbf{x}}_{i}
$$

where $\mathbf{w}_{k}$ is a column of $\mathbf{W}^{*}$. Constraining the source to $E\left\{s_{i k} s_{i k}^{*}\right\}=1$, the weights to $\|\mathbf{w}\|^{2}=1$, and $\mathbf{W}$ unitary due to the whitening transform [29].

The optimal weights $\mathbf{w}_{\text {opt }}$ are determined by maximizing the cost under the unit norm constraint where $\tilde{\mathbf{x}}_{i}$ has been whitened.

$$
\mathbf{w}_{\text {opt }}=\arg \max _{\|\mathbf{w}\|^{2}=1} E\left\{\left|G\left(\mathbf{w}^{*} \tilde{\mathbf{x}}_{i}\right)\right|^{2}\right\}
$$

where the nonlinearity $G$ : $\mathbb{C} \mapsto \mathbb{C}$. A number of possibilities exist for $G$ in (13), e.g., $G(\cdot)=\operatorname{acosh}(\cdot)$ or $G(\cdot)=\sin (\cdot)$.

To calculate the optimal weights, a gradient optimization algorithm is used, followed by a normalization step.

$$
\begin{aligned}
& \mathbf{w} \leftarrow \mathbf{w}+\mu \nu \frac{\partial J\left(s_{i}\right)}{\partial \mathbf{w}}, \\
& \mathbf{w} \leftarrow \frac{\mathbf{w}}{\|\mathbf{w}\|},
\end{aligned}
$$

where $J\left(s_{i}\right)=E\left\{\left|G\left(s_{i}\right)\right|^{2}\right\}, s_{i}=\mathbf{w}^{*} \tilde{\mathbf{x}}_{i}, \mu$ is the learning rate, and $\nu \in\{-1,1\}$ is the parameter that determines whether we are maximizing or minimizing the cost function.

Theorem 4.1: Let $J=J\left(s_{i}\right)$. Suppose $\mathbf{w}=\mathbf{w}^{R}+j \mathbf{w}^{I}$, and $\mathbf{w}^{R}$ and $\mathbf{w}^{I}$ are two real variables. The partial derivative of the cost function $J$ with respect to the conjugate of the weight vector $\mathbf{w}$, referblack to as the conjugate gradient, is obtained as

$$
\frac{\partial J}{\partial \mathbf{w}}=\frac{\partial J}{\partial \mathbf{w}^{R}}+j \frac{\partial J}{\partial \mathbf{w}^{I}}=E\left\{\tilde{\mathbf{x}}_{i} G^{*}\left(s_{i}\right) g\left(s_{i}\right)\right\},
$$

where $g$ is the derivation of $G$.

Proof: See Appendix A.

After each source is estimated, the vectors $\mathbf{w}$ are orthogonalized to prevent multiple solutions from converging to the same maximum since $\mathbf{W}$ is unitary due to the prewhitening step.

The mixed matrix is estimated as $\mathbf{A}=\mathbf{U}^{-1} \mathbf{W}^{*}$. We then recover the underlying sources by $\mathbf{S}=\mathbf{A}^{-1} \tilde{\mathbf{X}}$. Finally, the recoveblack underlying sources are normalized by the $\ell_{2}$ norm of $\mathbf{S}$, denoted as $\hat{\mathbf{S}}=\left[\hat{\mathbf{s}}_{1}, \hat{\mathbf{s}}_{2}, \ldots, \hat{\mathbf{s}}_{M}\right]^{T}$. The proposed algorithm is summarized in Algorithm 1. 


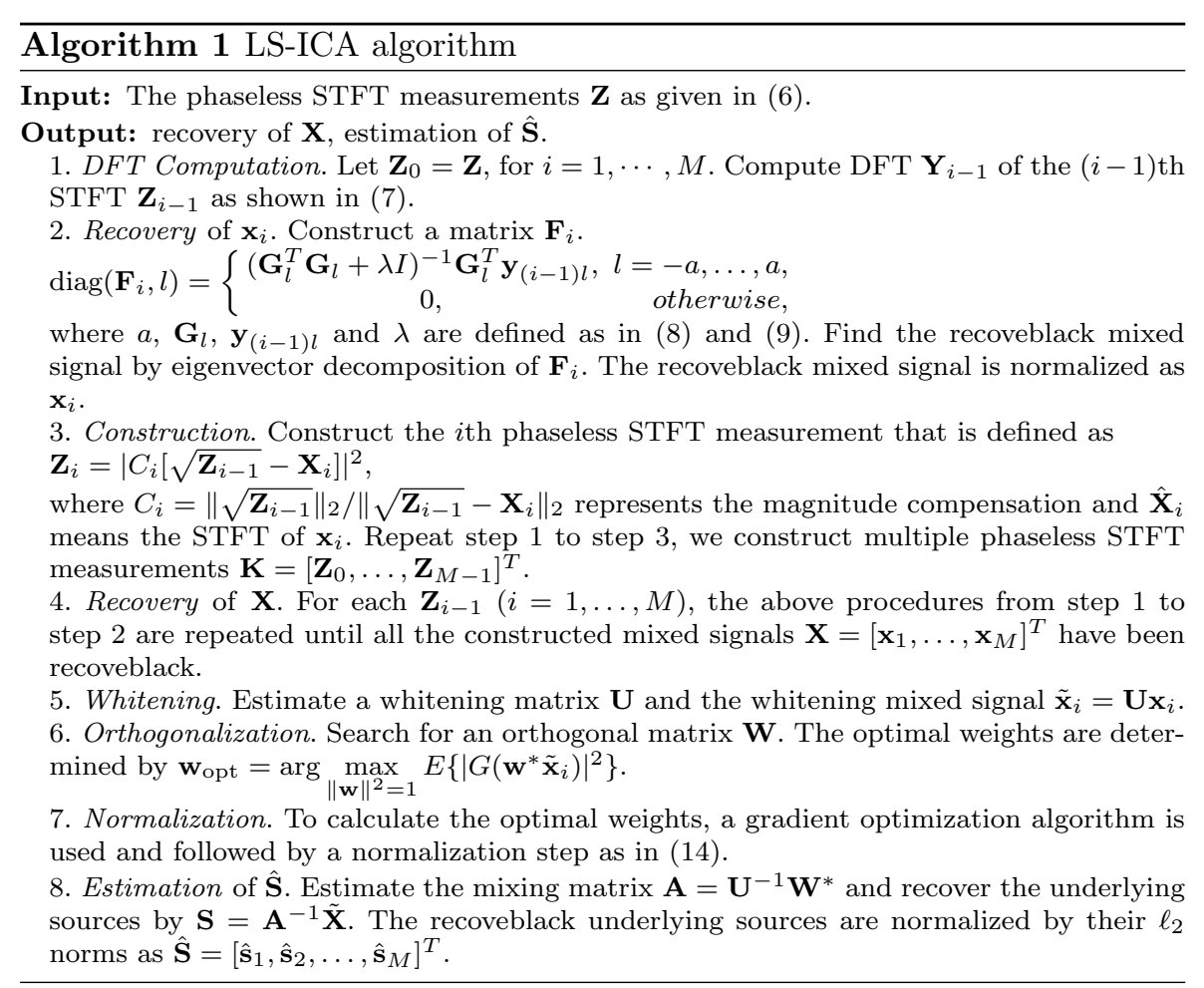

\subsection{GD-ICA algorithm}

When the conditions for the LS-ICA algorithm are not met (the maximal overlapping between adjacent windows $L>1$ and not long enough window length $W$ ), we present a GD-ICA algorithm for solving MISO phase retrieval problem.

(1) Recovery of the phases of the mixed signal $\mathbf{x}$

For $i=1$, let $\mathbf{Z}_{0}=\mathbf{Z}$ and $\mathbf{x}_{1}=\mathbf{x}$, DFT is used in the phaseless STFT measurement $\mathbf{Z}_{i-1}$ to obtain $\mathbf{Y}_{i-1}$ which is shown in (7).

For $L \leq W \leq\lceil N / 2\rceil$, let $\mathbf{D}_{\tau L} \in \mathbb{R}^{N \times N}$ be a diagonal matrix composed of the entries of $\mathbf{g}_{\tau L}$, the problem of recovering $\mathbf{x}_{i}$ from the measurement $\mathbf{Z}_{i-1}$ can therefore be equivalently posed as a non-convex loss function derived from

$$
f\left(\mathbf{x}_{i}\right)=\frac{1}{2} \sum_{\tau=0}^{R-1} \sum_{l=-a}^{a}\left(\mathbf{x}_{i}^{*} \mathbf{H}_{\tau, l} \mathbf{x}_{i}-Y_{i-1}(\tau, l)\right)^{2}
$$

where $\mathbf{H}_{\tau, l}$ and $\mathbf{x}_{i}^{*} \mathbf{H}_{\tau, l} \mathbf{x}_{i}$ are defined as in (5), and $a$ is defined in (8).

A GD algorithm is adopted to recover the $i$ th mixed signal by minimizing (16). The $k$ th iteration is 


$$
\begin{aligned}
& \mathbf{x}_{i k}=\mathbf{x}_{i(k-1)}-\mu \nabla f\left(\mathbf{x}_{i(k-1)}\right), \\
& \nabla f(\mathbf{b})=\sum_{\tau=0}^{R-1} \sum_{l=-a}^{a}\left(\mathbf{b}^{T} \mathbf{H}_{\tau, l} \mathbf{b}-Y_{i-1}(\tau, l)\right)\left[\left(\mathbf{H}_{\tau, l}+\mathbf{H}_{\tau, l}^{T}\right) \mathbf{b}\right],
\end{aligned}
$$

where $\mathbf{b}=\mathbf{x}_{i(k-1)}, \mu$ is the gradient step size, and $R$ is defined as in (1).

According to the above procedures, $\mathbf{x}_{i}$ is recoveblack from $\mathbf{Z}_{i-1}$.

(2) Construction of multiple mixed signals $\mathbf{X}$

This step is similar to the LS-ICA algorithm. In this step, we construct multiple mixed phaseless STFT measurements $\mathbf{K}$ by the residuals defined as in (10). The above procedures are repeated until all the constructed mixed signals are recoveblack from $\mathbf{K}$ and normalized as $\mathbf{X}=\left[\mathbf{x}_{1}, \ldots, \mathbf{x}_{M}\right]^{T}$.

(3) Recovery of multiple underlying sources $\mathbf{S}$

The CMN algorithm for complex-valued signals is used to recover the underlying sources from the normalized mixed signals $\mathbf{X}$. The recoveblack underlying sources are normalized as $\hat{\mathbf{S}}=\left[\hat{\mathbf{s}}_{1}, \hat{\mathbf{s}}_{2}, \ldots, \hat{\mathbf{s}}_{M}\right]^{T}$ to remove the impact of magnitude of the mixing matrix.

Improved loss function for initialization: For $L=1$, the study of Eldar et al. [4] shows that the geometry of the loss function for the initialization heavily affects the properties of the GD algorithm and the initialization $\mathbf{x}_{0}$ can be determined by a simple LS solution. However, it is prone to overfitting with less training data and requires the rank restriction of $\mathbf{G}_{l}$. Thus we construct a matrix $\mathbf{F}_{i 0}$ from (8) as follows

$$
\begin{aligned}
& \operatorname{diag}\left(\mathbf{F}_{i 0}, l\right)=\left\{\begin{array}{cl}
\left(\mathbf{G}_{l}^{T} \mathbf{G}_{l}+\lambda I\right)^{-1} \mathbf{G}_{l}^{T} \mathbf{y}_{(i-1) l}, & l=-a, \ldots, a, \\
0, & \text { otherwise, }
\end{array}\right. \\
& \text { subject to } \mathbf{F}_{i 0}=\mathbf{x}_{i 0} \mathbf{x}_{i 0}^{*},
\end{aligned}
$$

where $\mathbf{G}_{l}$ and $\mathbf{y}_{(i-1) l}$ are represented in (4), $a$ is defined as in (8), and $\mathbf{x}_{i 0}$ is a principle eigenvector of $\mathbf{F}_{i 0}$. Then the initialization $\mathbf{x}_{i 0}$ of the proposed GD algorithm can be constructed by $\mathbf{F}_{i 0}$.

In the case that $L>1$, we need to expand $\mathbf{y}_{(i-1) l}=\left\{Y_{i-1}(\tau, l)\right\}_{\tau=0}^{R-1}$ to an up-sampled version $\tilde{\mathbf{y}}_{(i-1) l}$ by expansion and interpolation as

$$
\begin{aligned}
& Y_{i-1}(n, l)= \begin{cases}Y_{i-1}(\tau, l), & n=\tau L, \\
0, & \text { otherwise, }\end{cases} \\
& \text { Let } \hat{\mathbf{y}}_{(i-1) l}=\left\{Y_{i-1}(n, l)\right\}_{n=0}^{N-1} \text { for fixed } l, \\
& \tilde{\mathbf{y}}_{(i-1) l}=\left(\mathbf{F}_{p}^{*} \mathbf{F}_{p}\right) \hat{\mathbf{y}}_{(i-1) l},
\end{aligned}
$$

where $\mathbf{F}_{p}$ is a partial Fourier matrix consisting of the first $R$ rows of the DFT matrix $\mathbf{F}$ defined as in (4). Then the initialization $\mathbf{x}_{i 0}$ can be obtained by (18).

The proposed algorithm is summarized in Algorithm 2. 


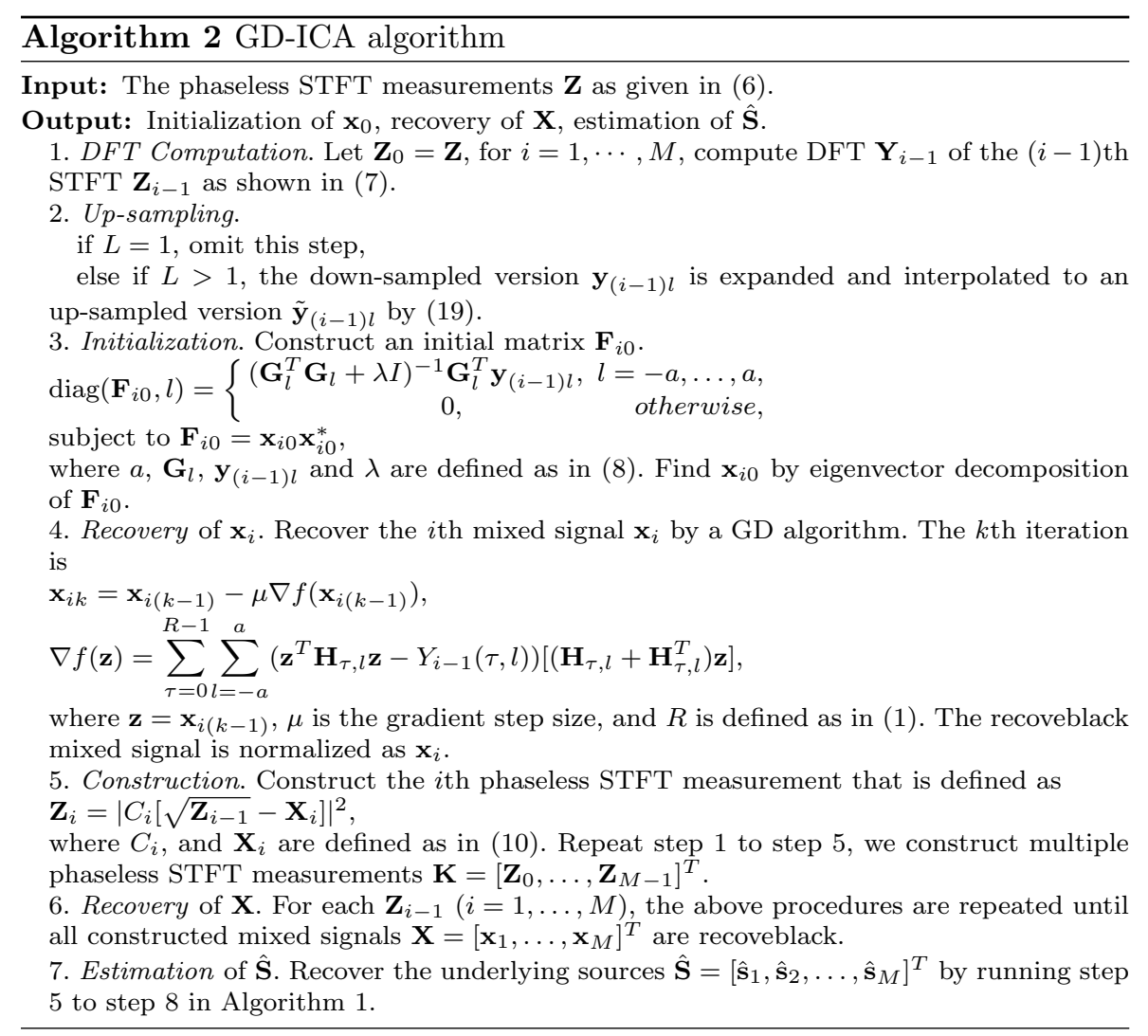

\section{Numerical Experiments}

In this section, we carry out numerical simulations on both synthetic and real source signals to demonstrate how the performance of the proposed algorithms depends on the length of the window and the maximal overlapping between adjacent windows, and how the algorithm is affected by noise for solving the problem of MISO phase retrieval.

The following experiments show that the LS-ICA algorithm estimates the phase of multiple sources and the mixing information if the window is sufficiently long and the maximal overlapping between adjacent windows $(L=1)$. When the window is shorter than or equal to $\lceil N / 2\rceil$, (8) does not have an improved LS solution. In this situation, the phase of multiple sources and the mixing information are recoveblack by the GD-ICA algorithm. In order to initialize the GD algorithm, we use a LS algorithm with a penalty term as described in section 4 .

The relative root mean squablack error (RRMSE) and signal to noise ratio (SNR) are used to evaluate the performance of the proposed algorithms. We consider two-source scenario. For $i=1,2, \mathrm{RRMSE}_{i}$ is defined as follows 


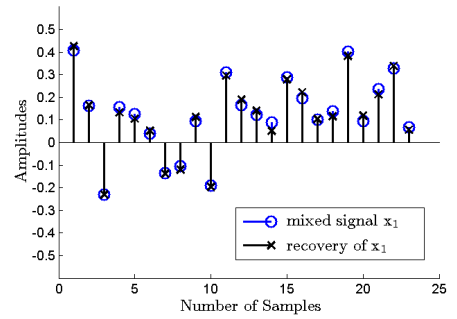

(a) Recovery of the mixed signal $\mathbf{x}_{1}$

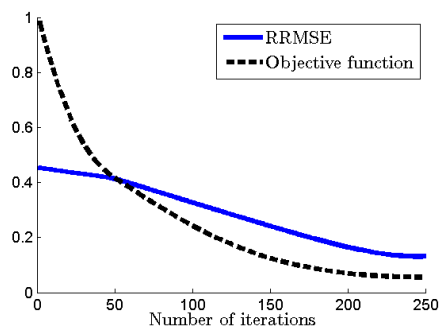

(c) RRMSE and the normalized objective function values of the recoveblack mixed signal $\mathbf{x}$

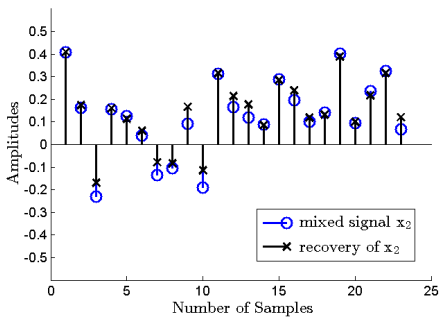

(b) Recovery of the constructed mixed signal $\mathbf{x}_{2}$

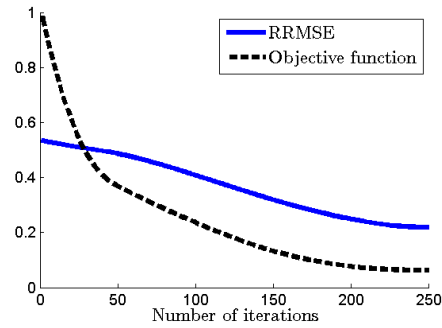

(d) RRMSE and the normalized objective function values of the recoveblack constructed mixed signal $\mathbf{x}_{2}$

Fig. 4: Recovery of the mixed signals (length $N=23$ ) with a rectangular window in a noisy environment of SNR $=25 \mathrm{~dB}$.

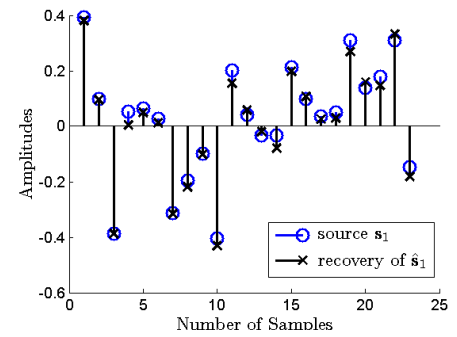

(a) Recovery of the underlying source $\hat{\mathbf{s}}_{1}$

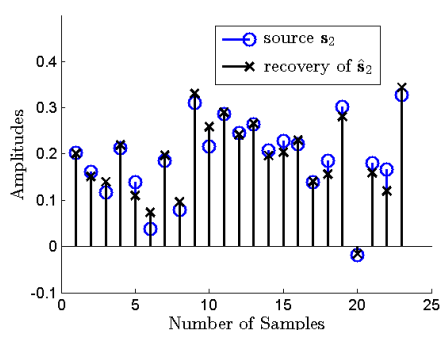

(b) Recovery of the underlying source $\hat{\mathbf{s}}_{2}$

Fig. 5: Recovery of the underlying sources (length $N=23$ ) with a rectangular window in a noisy environment of SNR $=25 \mathrm{~dB}$.

$$
\operatorname{RRMSE}_{i}=\frac{\operatorname{RMS}\left(\mathbf{s}_{i}-\hat{\mathbf{s}}_{i}\right)}{\operatorname{RMS}\left(\mathbf{s}_{i}\right)} .
$$


where $\hat{\mathbf{s}}_{i}$ is the recoveblack underlying source and $\mathbf{s}_{i}$ is the original source signal.

5.1 MISO phase retrieval from a synthetic phaseless STFT measurement

The underlying sources are randomly drawn and statistically independent. The mixed measurement is corrupted by additive Gaussian noise with zero mean and unit variance and the level of noise from $5 \mathrm{~dB}$ to $25 \mathrm{~dB}$.

In the first set of simulations, we evaluate the estimation performance of the proposed algorithm described in Algorithm 2. We choose the maximal overlapping between adjacent windows $(L=2)$, the window length $(W=10)$, the signal length $(N=23)$, the noise at $\mathrm{SNR}=25 \mathrm{~dB}$, and the number of short-time windows is denoted by $R=\lceil N / L\rceil$. The gradient step size $\mu$ is set to be 0.005 , the regularization coefficient $\lambda$ is set to be 0.01 , the maximal number of iterations for stopping Algorithm 2 is 3000. As shown in Fig. 4, the final RRMSEs of the recoveblack mixed signals $\mathbf{X}=\left[\mathbf{x}_{1}, \mathbf{x}_{2}\right]^{T}$ are less than 0.15 . Fig. 5 shows that the estimated underlying signals are similar to the source signals. The final RRMSEs of recoveblack source signals $\hat{\mathbf{s}}_{1}$ and $\hat{\mathbf{s}}_{2}$ are 0.1203 and 0.1022 which demonstrates the effectiveness of the proposed algorithm in recovering phases of multiple underlying signals.

The second experiment is to evaluate the performance of the proposed algorithm for the signal length $(N=43)$ in accordance with maximal overlapping between adjacent windows $L$. We choose the window length $(W=10)$, the noise at $\mathrm{SNR}=25 \mathrm{~dB}$, and the number of short-time windows is denoted by $R=\lceil N / L\rceil$. Fig. 6 illustrates the average final RRMSE for 45 experiments of the recoveblack mixed signals $\mathbf{X}=\left[\mathbf{x}_{1}, \mathbf{x}_{2}\right]^{T}$ and the recoveblack underlying sources $\hat{\mathbf{S}}=\left[\hat{\mathbf{s}}_{1}, \hat{\mathbf{s}}_{2}\right]^{T}$ for different $L$. For low values of $L=1,2$, the RRMSEs of $\mathbf{X}$ and $\mathbf{S}$ are relatively low. It shows clearly that the underlying sources can be recoveblack when the RRMSEs are smaller than 0.1. For high values of $L=3,4,5$, the RRMSEs of $\mathbf{X}$ are less than the RRMSEs of $\hat{\mathbf{S}}$ and the values are above 0.4 . These experimental results indicate that the interpolation is effective in up-sampling measurements especially for low values of $L$. The proposed algorithm has better recovery performance in $\hat{\mathbf{S}}$ than $\mathbf{X}$ for low values of $L$.

The third experiment is conducted to estimate the performance of the proposed algorithm with respect to different SNRs for the signal length $(N=$ 43). We choose the maximal overlapping between adjacent windows $(L=2)$, the window length $(W=10)$, and the number of short-time windows is denoted by $R=\lceil N / L\rceil$. Fig. 7 demonstrates the average RRMSE for 45 experiments of the recoveblack mixed signals $\mathbf{X}=\left[\mathbf{x}_{1}, \mathbf{x}_{2}\right]^{T}$ and the recoveblack underlying sources $\hat{\mathbf{S}}=\left[\hat{\mathbf{s}}_{1}, \hat{\mathbf{s}}_{2}\right]^{T}$ for different SNR. With the increase in SNR, the RRMSEs of $\mathbf{X}$ decrease slightly whereas the RRMSEs of $\hat{\mathbf{S}}$ drop rapidly. For SNR = $20 \mathrm{~dB}, 25 \mathrm{~dB}$, the RRMSEs of $\hat{\mathbf{S}}$ are relatively low and less than 0.15 . The results mean that the proposed algorithm has better anti-noise performance in $\hat{\mathbf{S}}$ than $\mathrm{X}$ especially for high values of SNR. 


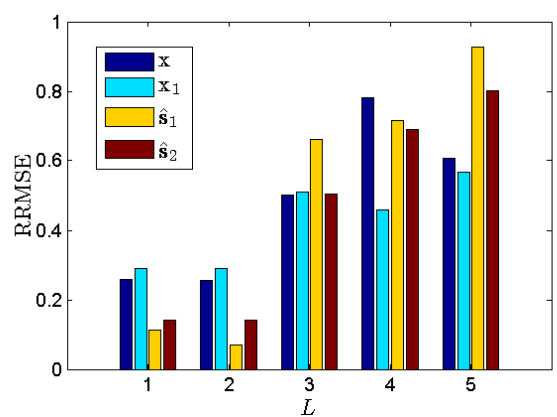

Fig. 6: The average final RRMSE for 45 experiments of the recoveblack mixed signals $\mathbf{X}$ and the recoveblack underlying sources $\hat{\mathbf{S}}$ for different $L$.

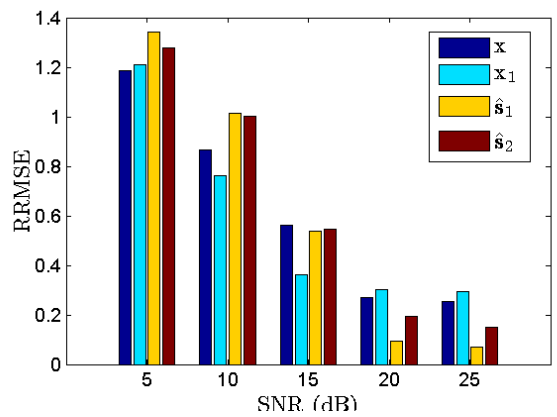

Fig. 7: The average final RRMSE for 45 experiments of the recoveblack mixed signals $\boldsymbol{X}$ and the recoveblack underlying sources $\hat{\mathbf{S}}$ for different SNR.

The fourth experiment aims to evaluate the recovery performance of the proposed algorithm for the signal length $(N=53)$, the maximal overlapping between adjacent windows $(L=1)$ and the noise $(\mathrm{SNR}=25 \mathrm{~dB})$ in accordance with the window length $W$. Fig. 8 and Fig. 9 show the estimation performance of the GD-ICA algorithm and the LS-ICA algorithm, respectively. Fig. 8 demonstrates the average final RRMSE for 40 experiments of the recoveblack mixed signals $\mathbf{X}=\left[\mathbf{x}_{1}, \mathbf{x}_{2}\right]^{T}$ and the recoveblack underlying sources $\hat{\mathbf{S}}=\left[\hat{\mathbf{s}}_{1}, \hat{\mathbf{s}}_{2}\right]^{T}$, and the RRMSEs of $\mathbf{X}$ and $\hat{\mathbf{S}}$ are relative low and less than 0.2. For $W>27$, the RRMSEs of $\mathbf{X}$ and $\hat{\mathbf{S}}$ increase rapidly. In Fig. 9, with the increasing of $W$, the RRMSEs of $\mathbf{X}$ and $\hat{\mathbf{S}}$ decrease sharply. For high values of $W>27$, the RRMSEs of $\mathbf{X}$ and $\hat{\mathbf{S}}$ are less than 0.3. These experiments demonstrate that the LS-ICA algorithm is effective in recovering the phases of multiple underlying signals when $W$ is long enough. When $W$ is not sufficiently long, the GD-ICA algorithm can recover the phases of multiple underlying signals effectively. 


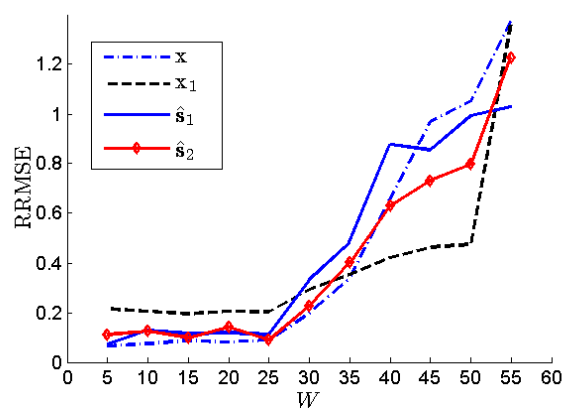

Fig. 8: GD-ICA algorithm: The average final RRMSE for 40 experiments of the recoveblack mixed signals $\mathbf{X}$ and the recoveblack underlying sources $\hat{\mathbf{S}}$ for different $W$.

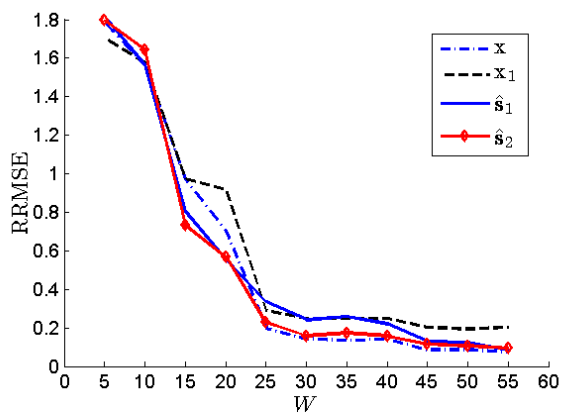

Fig. 9: LS-ICA algorithm: The average final RRMSE for 40 experiments of the recoveblack mixed signals $\mathbf{X}$ and the recoveblack underlying sources $\hat{\mathbf{S}}$ for different $W$.

\subsection{MISO phase retrieval for fetal ECG detection}

The maternal cutaneous electrode recordings normally contain fetal ECG signals (FECG) and maternal ECG signals (MECG). In this case, the inhibition of MECG and simultaneously detection of FECG are often expected [25]. However, sometimes the phases of the ECG recordings may be corrupted by noises, or the phaseless ECG recordings need to be transferblack for information hiding purposes.

Taking the fetal ECG detection as an example, the extraction of a FECG and a MECG from the abdominal mixed phaseless STFT measurement is chosen. The ECG data was obtained from MIT-BIH (http://www.physionet.org/cgibin/atm/ATM). We choose the signal length $(N=1000)$, the maximal overlapping between adjacent windows $(L=3)$, the window length $(W=10)$, the noise at $\mathrm{SNR}=25 \mathrm{~dB}$, and the number of short-time windows is denoted by $R=\lceil N / L\rceil$. 


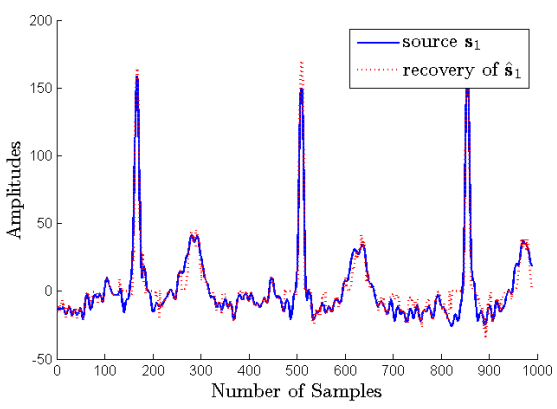

(a) Recovery of the underlying FECG signal $\hat{\mathbf{s}}_{1}$

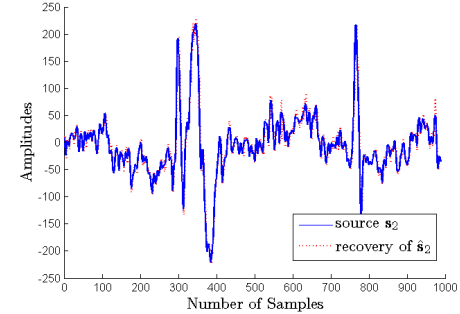

(b) Recovery of the underlying MECG signal $\hat{\mathbf{s}}_{2}$

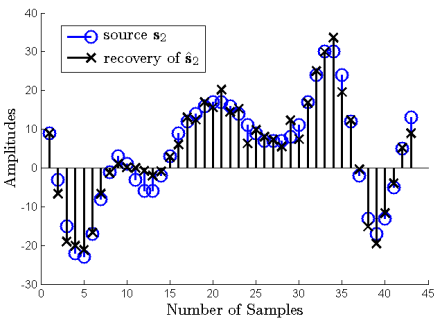

(d) The first 43 samples of the recoveblack MECG signal $\hat{\mathbf{s}}_{2}$ and the underlying MECG signal $s_{2}$ (c) The first 43 samples of the recoveblack FECG signal $\hat{\mathbf{s}}_{1}$ and the underlying FECG signal $s_{1}$

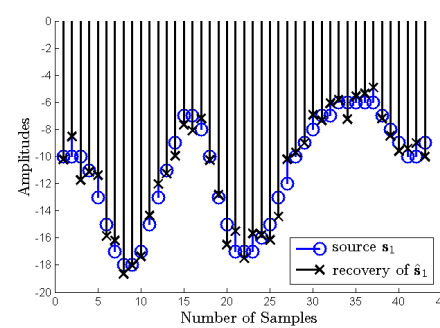

ig. 10: Recovery of the underlying signals (length $N=1000$ ) with a rectangular window in a noisy environment of $\mathrm{SNR}=25 \mathrm{~dB}$.
In [4], although the phase retrieval of a short underlying signal $(N \leq 50)$ from the phaseless STFT measurement has been studied, recovering phase information of a long underlying signal $(N>50)$ from the phaseless STFT measurement is limited by high errors. In order to improve the recovery precision of the relatively long signals, we transform the long signals to the matrix constructed by multiple short signals. The gradient step size $\mu$ is set to be 0.005 , the regularization coefficient $\lambda$ is set to be 0.01 , the maximal number of iterations for stopping the LS-ICA algorithm, the GD-ICA algorithm and the STFTMPR algorithm are 10000, 10000 and 20000 respectively. Table 1, 2 and 3 show clearly that the GD-ICA algorithm and the LS-ICA algorithm outperform the STFTMPR algorithm proposed in [4] with the maximal overlapping between adjacent windows $(L=3)$, the noise at $\mathrm{SNR}=25 \mathrm{~dB}$, and the window length $(W=10)$ in accordance with the signal length $N$.

As shown in Fig. 10, the estimated FECG signal $\hat{\mathbf{s}}_{1}$ and the estimated MECG signal $\hat{\mathbf{s}}_{2}$ are similar to the underlying FECG signal $\mathbf{s}_{1}$ and the underlying MECG signal $\mathbf{s}_{2}$. The final RRMSEs of the recoveblack FECG signal $\hat{\mathbf{s}}_{1}$ and the recoveblack MECG signal $\hat{\mathbf{s}}_{2}$ are 0.2548 and 0.1651 which demon- 
Table 1: The run time of LS-ICA algorithm, GD-ICA algorithm and STFTM$\mathrm{PR}$ algorithm in accordance with the signal length $N$.

\begin{tabular}{|c|c|c|c|}
\hline \multirow[t]{2}{*}{ Signal length $N$} & \multicolumn{3}{|c|}{ Run time/s } \\
\hline & LS-ICA & GD-ICA & STFTMPR \\
\hline 23 & 1.9503 & 1.987 & 2.0016 \\
\hline 100 & 3.8016 & 3.2145 & 16.7637 \\
\hline 150 & 4.9221 & 4.0657 & 71.1252 \\
\hline 200 & 8.4453 & 8.1133 & 86.9734 \\
\hline 350 & 10.8176 & 10.6347 & 107.1148 \\
\hline 500 & 29.9661 & 27.7732 & 408.3776 \\
\hline 650 & 29.6387 & 28.3128 & 557.4145 \\
\hline 800 & 41.9241 & 39.5589 & 963.4996 \\
\hline 1000 & 43.0489 & 41.9765 & 1685.3366 \\
\hline
\end{tabular}

Table 2: The RRMSE of LS-ICA algorithm, GD-ICA algorithm and STFTM$\mathrm{PR}$ algorithm in accordance with the signal length $N$.

\begin{tabular}{cccc}
\hline Signal length $N$ & & RRMSE & \\
& LS-ICA & GD-ICA & STFTMPR \\
\hline 23 & 0.1171 & 0.118 & 0.1107 \\
100 & 0.1078 & 0.1012 & 0.1098 \\
150 & 0.112 & 0.0998 & 0.1764 \\
200 & 0.1207 & 0.1087 & 0.1953 \\
350 & 0.1277 & 0.1209 & 0.2999 \\
500 & 0.151 & 0.149 & 0.3095 \\
650 & 0.1486 & 0.1301 & 0.3318 \\
800 & 0.1168 & 0.1175 & 0.4185 \\
1000 & 0.1455 & 0.1406 & 0.4378 \\
\hline
\end{tabular}

Table 3: The iteration number of LS-ICA algorithm, GD-ICA algorithm and STFTMPR algorithm in accordance with the signal length $N$.

\begin{tabular}{|c|c|c|c|}
\hline \multirow[t]{2}{*}{ Signal length $N$} & \multicolumn{3}{|c|}{ Iteration number } \\
\hline & LS-ICA & GD-ICA & STFTMPR \\
\hline 23 & 524 & 515 & 403 \\
\hline 100 & 727 & 679 & 1570 \\
\hline 150 & 999 & 847 & 3284 \\
\hline 200 & 1681 & 1530 & 3539 \\
\hline 350 & 2075 & 1911 & 3463 \\
\hline 500 & 6112 & 5398 & 7736 \\
\hline 650 & 5824 & 6023 & 9645 \\
\hline 800 & 6873 & 6544 & 11967 \\
\hline 1000 & 8207 & 8021 & 18408 \\
\hline
\end{tabular}


strates the efficiency of the proposed algorithm in recovering the phases of real FECG and MECG signals.

\section{Conclusion}

The problem of multi-source phase retrieval from single channel mixed phaseless STFT measurement has been investigated in this paper.

We have formed a new model for the problem of multi-source phase retrieval from a mixed phaseless STFT measurement.

For maximal overlap between adjacent windows $(L=1)$ and sufficiently long window length $W$, we propose an LS-ICA algorithm. When the window length $W$ is not long enough, a GD-ICA algorithm is presented as a solution to this problem.

We have also shown the significance of the initialization method to the GD-ICA algorithm and demonstrated that the initialization method can be constructed by an improved LS loss function.

Numerical experiments show that the proposed algorithms perform well in estimating the phase of multiple sources and the mixing information. In terms of future research directions, it is interesting to investigate how to incorporate conditions such as window length, additional magnitude-only measurement, mixed model (e.g. linear mixed model, nonlinear mixed model), or maximal overlapping between adjacent windows into the MISO phase retrieval algorithms. It would also be interesting to extend the consideblack model and proposed methods to other scenarios such as for single input single output (SISO) system and multiple input multiple output (MIMO) system.

\section{Acknowledgements}

This work was supported by the National Natural Science Foundation of China under Grant 61301250, the Key Research and Development Project of Shanxi Province under Grant 201803D421035, the Outstanding young academic leaders of Higher Learning Institutions of Shanxi under Grant [2015]3 and the Outstanding Innovative Teams of Higher Learning Institutions of Shanxi under Grant 201705D131025. The work was conducted during Guo's visit at the University of Surrey.

\section{Appendix}

\section{A. Proof of Theorem 4.1}

The derivative of the function $J=J\left(s_{i}\right)$ is calculated based on real-valued functions because $J$ is not analytic. Let $s_{i}=\mathbf{w}^{*} \tilde{\mathbf{x}}_{i}, G\left(s_{i}\right)$ is expended in terms 
of two real-valued functions $u(y)$ and $v(y)$.

$$
\begin{aligned}
J & =\left|G\left(s_{i}\right)\right|^{2}=\left|G\left(\mathbf{w}^{*} \tilde{\mathbf{x}}_{i}\right)\right|^{2}=\left|u\left(\mathbf{w}^{*} \tilde{\mathbf{x}}_{i}\right)+j v\left(\mathbf{w}^{*} \tilde{\mathbf{x}}_{i}\right)\right|^{2} \\
& \equiv u^{2}(a, b)+v^{2}(a, b)
\end{aligned}
$$

where $a, b$ are the real part and the imaginary part of $\mathbf{w}^{*} \tilde{\mathbf{x}}_{i}$ respectively. The partial derivative of $J$ with respect to the real weight $w_{i}^{R}$ is obtained by use of the chain rule and results in

$$
\frac{\partial J}{\partial w_{i}^{R}}=2 u\left(\frac{\partial u(a, b) \partial a}{\partial a \partial w_{i}^{R}}+\frac{\partial u(a, b) \partial b}{\partial b \partial w_{i}^{R}}\right)+2 v\left(\frac{\partial u(a, b) \partial a}{\partial a \partial w_{i}^{R}}+\frac{\partial u(a, b) \partial b}{\partial b \partial w_{i}^{R}}\right) .
$$

The above expression is rearranged as follows

$$
\begin{aligned}
\frac{\partial J}{\partial w_{i}^{R}} & =2 u\left(u_{a} \tilde{x}_{i j}^{R}+u_{b} \tilde{x}_{i j}^{I}\right)+2 v\left(v_{a} \tilde{x}_{i j}^{R}+v_{b} \tilde{x}_{i j}^{I}\right) \\
& =2\left[\tilde{x}_{i j}^{I}\left(u u_{a}+v v_{a}\right)+\tilde{x}_{i j}^{R}\left(u u_{b}+v v_{b}\right)\right] .
\end{aligned}
$$

where $u_{a} \equiv \frac{\partial u(a, b)}{\partial a}, u_{b} \equiv \frac{\partial u(a, b)}{\partial b}, v_{a} \equiv \frac{\partial v(a, b)}{\partial a}$, and $v_{b} \equiv \frac{\partial v(a, b)}{\partial b}$.

The derivative of $J$ with respect to the imaginary weight $w_{i}^{I}$ is calculated as

$$
\frac{\partial J}{\partial w_{i}^{I}}=2\left[\tilde{x}_{i j}^{I}\left(u u_{a}+v v_{a}\right)-\tilde{x}_{i j}^{R}\left(u u_{b}+v v_{b}\right)\right] .
$$

It is advantageous to utilize complex operators for a more compact notation. Noticing that

$$
\left(u u_{a}+v v_{a}\right)+j\left(u u_{b}+v v_{b}\right)=g^{*}\left(\mathbf{w}^{*} \tilde{\mathbf{x}}_{i}\right) G\left(\mathbf{w}^{*} \tilde{\mathbf{x}}_{i}\right),
$$

where $g$ is the derivative of $G$. According to the Cauchy-Riemann equations: $g^{R}=u_{a}=v_{b}$ and $g^{I}=v_{a}=-u_{b}$, the derivative of $J$ with respect to the complex weight vector $\mathbf{w}$ is shown as:

$$
\frac{\partial J}{\partial \mathbf{w}}=2\left(g^{*}\left(\mathbf{w}^{*} \tilde{\mathbf{x}}_{i}\right) G\left(\mathbf{w}^{*} \tilde{\mathbf{x}}_{i}\right)\right)^{*} \tilde{\mathbf{x}}_{i}=2 \tilde{\mathbf{x}}_{i} G^{*}\left(\mathbf{w}^{*} \tilde{\mathbf{x}}_{i}\right) g\left(\mathbf{w}^{*} \tilde{\mathbf{x}}_{i}\right),
$$

which is equivalent to

$$
\frac{\partial J(\mathbf{w})}{\partial \mathbf{w}}=\frac{1}{2}\left(\frac{\partial J(\mathbf{w})}{\partial \mathbf{w}^{R}}+j \frac{\partial J(\mathbf{w})}{\partial \mathbf{w}^{I}}\right) .
$$

\section{References}

1. Ahmed, A., Recht, B., Romberg, J.: Blind deconvolution using convex programming. IEEE Trans. Inform. Theory 60(3), 1711-1732 (2014)

2. Al-Tmeme, A., Woo, W., Dlay, S., Gao, B.: Single channel informed signal separation using artificial-stereophonic mixtures and exemplar-guided matrix factor deconvolution. International Journal of Adaptive Control and Signal Processing (2018)

3. Baykal, B.: Blind channel estimation via combining autocorrelation and blind phase estimation. IEEE Trans. Circuits Syst. I: Regular Papers 51(6), 1125-1131 (2004) 
4. Bendory, T., Eldar, Y.C.: Non-convex phase retrieval from STFT measurements. IEEE Trans. Inf. Theory PP(99), 1-1 (2017)

5. Candes, E.J., Eldar, Y.C., Strohmer, T., Voroninski, V.: Phase retrieval via matrix completion. SIAM Review 57(2), 225-251 (2015)

6. Dashen, R., Flatté, S.M., Munk, W.H., Watson, K.M., Zachariasen, F.: Sound Transmission Through a Fluctuating Ocean. Cambridge University Press (2010)

7. Eldar, Y.C., Sidorenko, P., Mixon, D.G., Barel, S., Cohen, O.: Sparse phase retrieval from short-time Fourier measurements. IEEE Signal Process. Lett. 22(5), 638-642 (2015)

8. Epple, F.M., Ehn, S., Thibault, P., Koehler, T., Potdevin, G., Herzen, J., Pennicard, D., Graafsma, H., Noel, P.B., Pfeiffer, F.: Phase unwrapping in spectral x-ray differential phase-contrast imaging with an energy-resolving photon-counting pixel detector. IEEE Trans. Med. Imag. 34(3), 816-823 (2015)

9. Fienup, C., Dainty, J.: Phase retrieval and image reconstruction for astronomy. Image Recovery: Theory and Application pp. 231-275 (1987)

10. Fienup, J.R.: Phase retrieval algorithms: a comparison. Applied Optics 21(15), 2758$2769(1982)$

11. Fienup, J.R.: Phase-retrieval algorithms for a complicated optical system. Appl. Optics 32(10), 1737-1746 (1993)

12. Gerchberg, R.W.: A practical algorithm for the determination of phase from image and diffraction plane pictures. Optik 35(2), 237-246 (1972)

13. Gerkmann, T., Krawczyk Becker, M., Le Roux, J.: Phase processing for single-channel speech enhancement: History and recent advances. IEEE Signal Proc. Mag. 32(2), 55-66 (2015)

14. Griffin, D.W., Lim, J.S.: Signal estimation from modified shorttime Fourier transform. IEEE Trans. Acoust. Speech Signal Process. 32(2), 236-243 (1984)

15. Guo, Y., Huang, S., Li, Y.: Single-mixture source separation using dimensionality reduction of ensemble empirical mode decomposition and independent component analysis. Circ. Syst. Signal Pr. 31(6), 2047-2060 (2012)

16. Guo, Y., Huang, S., Li, Y., Naik, G.R.: Edge effect elimination in single-mixture blind source separation. Circ. Syst. Signal Pr. 32(5), 2317-2334 (2013)

17. Guo, Y., Wang, A., Wang, W.: Multi-source phase retrieval from multi-channel phaseless STFT measurements. Signal Processing 144, 36-40 (2018)

18. Huang, K., Eldar, Y.C., Sidiropoulos, N.D.: Phase retrieval from 1D Fourier measurements: Convexity, uniqueness, and algorithms. IEEE Trans. Signal Process. 64(23), 6105-6117 (2016)

19. Hyunjin, C., Junseok, L., Bonhwa, K., Cheong, M., Iksu, S., Hanseok, K., Wooyoung, H.: Underwater radiated signal analysis in the modulation spectrogram domain. IEICE Trans. Fund. Electr. 98(8), 1751-1759 (2015)

20. Hyvarinen, A., Karhunen, J., Oja, E.: Independent Component Analysis. John WileySons, New York, NY, USA (2004)

21. Jaganathan, K., Eldar, Y., Hassibi, B.: STFT phase retrieval: Uniqueness guarantees and recovery algorithms. IEEE J. Sel. Top. Signal Process. 10(4), 770-781 (2016)

22. Jaganathan, K., Eldar, Y.C., Hassibi, B.: Phase retrieval: An overview of recent developments. arXiv preprint arXiv:1510.07713 (2015, submitted for publication)

23. Krawczyk, M., Gerkmann, T.: STFT phase reconstruction in voiced speech for an improved single-channel speech enhancement. IEEE/ACM Trans. Audio Speech 22(12), 1931-1940 (2014)

24. Kulmer, J., Mowlaee, P.: Phase estimation in single channel speech enhancement using phase decomposition. IEEE Signal Process. Lett. 22(5), 598-602 (2015)

25. Liu, S., Li, Y., Hu, X., Liu, L., Hao, D.: A novel thresholding method in removing noises of electrocardiogram based on wavelet transform. Journal of Information \&Computational Science 10(15), 5031-5041 (2013)

26. Lyon, D.: The discrete Fourier transform, Part 6: Cross-correlation. Journal of Object Technology 9(2), 17-22 (2010)

27. Miao, J., Ishikawa, T., Robinson, I.K., Murnane, M.M.: Beyond crystallography: Diffractive imaging using coherent X-ray light sources. Science 348(6234), 530-535 (2015)

28. Novey, M., Adali, T.: ICA by maximization of nongaussianity using complex functions. In: IEEE Workshop Mach. Learn. Signal Process., pp. 21-26. IEEE (2005) 
29. Novey, M., Adali, T.: Complex ICA by negentropy maximization. IEEE Trans. Neural Networks 19(4), 596-609 (2008)

30. Parathai, P., Woo, W., Dlay, S., Gao, B.: Single-channel blind separation using L 1sparse complex non-negative matrix factorization for acoustic signals. The Journal of the Acoustical Society of America 137(1), EL124-EL129 (2015)

31. Patton, L.K., Rigling, B.D.: Phase retrieval for radar waveform optimization. IEEE Trans. Aero. Elec. Sys. 48(4), 3287-3302 (2012)

32. Rabiner, L.R., Juang, B.H.: Fundamentals of Speech Recognition. PTR Prentice Hall (1993)

33. Rajaei, B., Tramel, E.W., Gigan, S., Krzakala, F., Daudet, L.: Intensity-only optical compressive imaging using a multiply scattering material and a double phase retrieval approach. In: Proc. IEEE Int. Conf. Acoust., Speech, Signal Process., pp. 4054-4058. IEEE (2016)

34. Shechtman, Y., Eldar, Y.C., Cohen, O., Chapman, H.N., Miao, J., Segev, M.: Phase retrieval with application to optical imaging: a contemporary overview. IEEE Signal Process. Mag. 32(3), 87-109 (2015)

35. Tengtrairat, N., Gao, B., Woo, W., Dlay, S.: Single-channel blind separation using pseudo-stereo mixture and complex 2-d histogram. IEEE Trans. Neural Networks 24(11), 1722-1735 (2013)

36. Tengtrairat, N., Woo, W., Dlay, S., Gao, B.: Online noisy single-channel source separation using adaptive spectrum amplitude estimator and masking. IEEE Trans. Signal Process. 64(7), 1881-1895 (2016)

37. Woo, W., Gao, B., Bouridane, A., Ling, B., Chin, C.: Unsupervised learning for monaural source separation using maximization-minimization algorithm with time-frequency deconvolution. Sensors 18(5) (2018)

38. Xie, J., Lin, W., Li, H., Guo, K., Jin, B., Zhang, Y., Liu, D.: A new algorithm for improving deblurring effects and addressing spatially-variant blur problems for image motion deblurring. In: Proc. 4th Int. Cong. Image, Signal Process., vol. 2, pp. 651-655 (2011)

39. Zhang, C., Lin, W., Li, W., Zhou, B., Xie, J., Li, J.: Improved image deblurring based on salient-region segmentation. Image Commun. 28(9), 1171-1186 (2013). DOI 10.1016/j.image.2013.07.004. URL http://dx.doi.org/10.1016/j.image.2013.07.004

40. Zhang, F., Pedrini, G., Osten, W.: Phase retrieval of arbitrary complex-valued fields through aperture-plane modulation. Phys. Rev. A 75(4), 043805 (2007) 